\title{
The effectiveness of preoperative individual information on reducing anxiety and pain after hysterectomy: A randomized controlled trial
}

\author{
Hrønn Thorn*1, Lisbeth Uhrenfeldt ${ }^{2}$ \\ ${ }^{1}$ Oncological Outpatient Clinic, Vejle, Denmark \\ ${ }^{2}$ Faculty of Nursing and Health Science, Nord University, Norway
}

Received: April 3, 2019

DOI: $10.5430 /$ jnep.v10n1p67
Accepted: September 23, 2019

Online Published: September 27, 2019

URL: https://doi.org/10.5430/jnep.v10n1p67

\begin{abstract}
Background and objective: Preoperative anxiety among gynecological patients is well-known and has a direct correlation with postoperative pain. By minimizing preoperative anxiety, the level of postoperative pain may decrease. The purpose of this study was to evaluate the effect of preoperative structured information and dialogue on patients' anxiety and postoperative pain.

Methods: A single-center non-blinded randomized controlled trial. Forty-six women scheduled for hysterectomy were allocated either to the study group or the control group. The study group was given individual information at a preoperative consultation while the control group was given information at admittance. The main outcome was anxiety level and postoperative pain.

Results: Forty participants (study group $=20$; control group $=20$ ) were analyzed. No statistically significant difference was found in anxiety level within the first $24 \mathrm{~h}$ postoperatively or in postoperative pain within four weeks between the groups.

Conclusions: Preoperative individual information and dialogue did not result in significant effects in reducing anxiety level nor did it result in lower postoperative pain score.
\end{abstract}

Key Words: Anxiety, Clinical trial, Nursing, Postoperative pain

\section{INTRODUCTION}

Preoperative anxiety is experienced by many patients scheduled for various surgeries. ${ }^{[1-3]}$ The extent of preoperative anxiety is not entirely clear. According to Carr et al. ${ }^{[4]}$ preoperative anxiety is experienced by $11 \%-80 \%$ of patients. ${ }^{[4]}$ The patients experience psychological and physical stress, such as anxiety and fear of anesthesia and postoperative pain, fear of waking up during the surgery and loss of control, and hence, it is a problem that implicates postoperative recovery. Preoperative anxiety delays wound healing and increase the need for medication, increases blood pressure, increases body temperature and affects patients' well-being. ${ }^{[3]}$ According to patients, lack of information, the presence of illness and poor organization of the continuity of care contribute to preoperative anxiety. ${ }^{[4,5]}$

High standards of cost-effectiveness in healthcare systems have led to an accelerated continuity of care, in which the patient experiences many transitions in a short time and brief contacts with healthcare professionals. ${ }^{[3]}$ During these transitions the patient feels insignificant and without control. This lack of control has a negative impact on the patient's wellbeing and contributes to increased anxiety levels. For the healthcare providers, these brief patient contacts make it difficult to identify preoperative anxiety due to the timeframe. ${ }^{[3]}$

Preoperative anxiety among gynecological patients is more common than among other surgical patients. ${ }^{[4]}$ Having a hysterectomy typically causes stress and fear, which is con-

*Correspondence: Hrønn Thorn; Email: Hronn.Thorn@rsyd.dk; Address: Oncological Outpatient Clinic, Vejle, Denmark. 
sistent with the idea that a hysterectomy is associated with the loss of reproduction, femininity and attractiveness. ${ }^{[4,6]}$

Chronic postsurgical pain is a potential adverse event following all kinds of surgery. The cause of chronic postsurgical pain is multifactorial and some of the known risk factors are; female sex, acute postoperative pain, preexisting pain and anxiety. The incidence of chronic postsurgical pain after hysterectomy on benign indication is approximately $30 \%$. Having chronic pain affects the women's daily living and quality of life. ${ }^{[7,8]}$

Knowing that preoperative anxiety has a direct correlation with postoperative pain ${ }^{[2,7,9,10]}$ and that patients who experience severe postoperative pain can develop chronic postsurgical pain, it is of the utmost importance to prevent postoperative pain. ${ }^{[7,11]}$ By minimizing preoperative anxiety, patients' level of postoperative pain will decrease ${ }^{[4,12-14]}$ and experiences of well-being will increase ${ }^{[5]}$ along with patient satisfaction. ${ }^{[15,16]}$ Preoperative anxiety can be minimized by providing the patient with comprehensive information on the entire process, creating transparency and predictability. It is best to provide this comprehensive information before the day of the surgery, as patients' level of anxiety is most likely high at admission, and therefore, they may have difficulty receiving and understanding information. ${ }^{[15,17,18]}$

In a systematic review that updated evidence regarding preoperative education interventions and their effect on health outcomes among patients scheduled for elective cardiac surgery, only two of the included six studies demonstrated a significant difference in preoperative anxiety level in the intervention group. Also, with regards to postoperative pain, there was no significant difference between the groups in the two studies. ${ }^{[19]}$

Another review that included 14 trials assessed the effect of preoperative education on postoperative outcomes among cardiac patients. The finding from this review demonstrated that preoperative education reduced the anxiety level. The meta-analysis was based on nine trials of which three used Spielberger State-Trait Anxiety Index (STAI) to measure the level of anxiety. However, the review failed to demonstrate any statistically significant difference in postoperative pain. Only three trials were included in the meta-analysis that measured the level of postoperative pain. ${ }^{[20]}$

According to another review that included orthopedic patients, there was no statistically significant difference between the groups with regards to postoperative pain. However, there was a statistically significant difference in the level of preoperative anxiety. Three trials measured pain using VAS and were included in a meta-analysis. Four trials measured preoperative anxiety using STAI and were included in a meta-analysis. ${ }^{[21]}$

The conclusion of the three reviews with regards to postoperative pain, were based on low quality evidence. ${ }^{[19-21]}$

A newly published randomized controlled trial that included 82 patients undergoing spinal surgery found a significant difference in the level of anxiety and postoperative pain. In this study, anxiety was measured using the STAI score and pain was measured on a visual analog scale (VAS). Both groups were given educational intervention on the day before surgery and in addition, the intervention group received a booklet and individualized information that included videos and pictures. ${ }^{[13]}$

In summary, we know that gynecological patients are anxious before surgery, that transparency and predictability may lower the level of anxiety and that anxiety correlates directly with postoperative pain, ${ }^{[2]}$ but we do not know if preoperative information and dialogue before hysterectomy influence the level of postoperative pain after hysterectomy. Therefore, there is a need for more knowledge on this subject.

The purpose of this study was to evaluate the effect of preoperative structured information and dialogue on preoperative anxiety and postoperative pain after hysterectomy on benign indication. We also evaluated postoperative features such as nausea and vomiting, oral intake, activity and length of stay. The hypothesis was that preoperative structured information and dialogue decrease the level of anxiety and postoperative pain.

\section{Methods}

This study was a non-blinded, single center, randomized trial. The approval was obtained from the Ethics Committee of the Central Denmark Region (no. M2013-26313). The trial fulfilled the requirements of the Helsinki Declaration and was conducted in accordance with GCPICH guidelines. This trial was registered at the Danish Data Protection Agency and at Controlled-trials.com. URL http://www. isrctn. com/ISRCTN53835337.

\subsection{Setting and participants}

The study was carried out at a Danish public teaching hospital. The participants of this study were enrolled from November 2013 to June 2016.

Eligibility criteria for the participants were as follows: women, age $>18$ years, and scheduled for elective hysterectomy on benign indication. Exclusion criteria were women with anxiety neurosis and other mental disorders, daily preoperative use of anxiolytics and opioids, known allergy to opioids, chronic pain, no ability to communicate in Danish, 
and a body mass index $(\mathrm{BMI})>35$.

Before any study-related procedure was performed, the participants were given verbal and written information about the study and written and informed consent was obtained.

Potential participants were screened at the Gynecological Outpatient Clinic and by using logistics switch boards in the electronic patient record. Those patients likely to meet the inclusion criteria who were screened in the Gynecological Outpatient Clinic, received written and verbal information about the study, and those who were screened using the logistics switch board received a recruitment letter with information about the study and participant requirements. Enclosed in the recruitment letter was a preaddressed envelope and written informed consent to return with a signature to the investigator if the women decided to participate in the study.

\subsection{Study design}

Randomization with a block size of 8 and a ratio of $1: 1$ with sealed envelopes was used to assign the patients to; a preoperative meeting with individual information and dialogue, the study group (SG) or to standard treatment, the control group (CG). To avoid bias, the random list and preparing the envelopes according to the random list was done by an investigator without clinical involvement in the study. After randomization, all participants received a letter that told them which group they were assigned to, and for those in the SG, a preoperative visit was scheduled $14 \pm 7$ days before the surgery. The first author, who was responsible for collecting data, was not blinded.

\subsection{Standard information given to the control group}

The information provided was based on the pamphlet describing the current operation and was given to patients upon admission to the gynecological department on the day of the surgery. If the participant was the first patient to go to the theater, there was approximately 45 minutes to prepare the patient and provide information. If the patient was number two or three, there was usually more time to give information, depending on the workload in the ward.

\subsection{Intervention}

The intervention involved a 45-minute preoperative visit at the outpatient clinic approximately two weeks before the surgery. The information provided at the visit was given chronologically i.e. starting on the evening before surgery and ending with the completion of the recovery period. In addition to the information in the pamphlet, the information included what the participant would face upon arrival to the operating area, the characteristics of the operating theater, anesthetic procedures, what to expect upon waking up in the recovery room and the return to the gynecological department.

To enhance the SG self-efficacy, systematic evidence-based advice was given about how to manage postoperative pain, postoperative nausea and postoperative bowel function.

The participants were invited to talk about their worries and expectation. The information given to all participants in the SG was provided by the first author.

\subsection{Sample size}

The sample size of this study was based on the calculation of an expected difference of $35 \%$ in the number of patients with moderate to severe postoperative pain during the first $24 \mathrm{~h}$ postoperatively. Moderate to severe pain corresponds to a score $>6$ on a VAS. With $80 \%$ power $(\alpha=0.05, \beta=$ 0.20 ), a sample size of 36 patients per group was necessary. A conservative sample size of 80 was chosen to allow incomplete data collection. To recruit this number of patients, the inclusion period was set to 25 months and later prolonged by 12 additional months.

\subsection{Data collection}

To evaluate the impact of preoperative information and dialogue, the following well-validated instruments were used: the State Trait Anxiety Inventory questionnaire (STAI), ${ }^{[22]}$ the McGill Short-Form Pain Questionnaire (SF-MPQ), ${ }^{[23]}$ and a VAS $^{[24]}$ to measure the intensity of nausea. The STAI questionnaire evaluates the current level of anxiety and consists of 20 items on a 4-point Likert scale. The cut-off points are as follows: mild anxiety, 20-39; moderate anxiety, 4059; and high anxiety, 60-80. The SF-MPQ consists of three items: one with 18 words (Pain Rating Index [PRI]), one presenting Present Pain Intensity (PPI), and one with a VAS, which is a horizontal line $100 \mathrm{~mm}$ in length (where $0=$ no pain, $100=$ worst imaginable pain). Anxiety level was assessed upon admission on the day of the surgery, $6 \mathrm{~h}$ and 24 $\mathrm{h}$ after surgery. Assessment of nausea was measured $6 \mathrm{~h}$ and $24 \mathrm{~h}$ after surgery, and pain was measured at $6 \mathrm{~h}$ and $24 \mathrm{~h}$ after surgery and again at 4-week follow-up. According to clinical standards, the cut-off point for measuring moderate to severe pain on a VAS was $>6$.

Requirements for rescue antiemetic and morphine during the first $24 \mathrm{~h}$ postoperative were documented in patients' medical journals.

Assessments of anxiety, pain and nausea were completed by the participants themselves and noted in a diary as well as data about oral intake, activity and vomiting.

In the case the participants were discharged before $24 \mathrm{~h}$ follow-up, they received a stamped envelope to return the 
diary.

Four weeks after surgery, the participants filled in the SFMPQ and returned the questionnaire in a stamped envelope.

The primary endpoint with respect to efficacy on pain was the proportion of patients with a VAS score $<6$. Secondary endpoints were level of anxiety, nausea, oral intake, vomiting, activity and postoperative length of stay.

\subsection{Data Analysis}

Statistical analysis was performed using STATA 10 (StataCorp Texas, USA), and EpiData version 3.1 (EpiData Association, Odense, Denmark) was used for data entry. Data were analyzed using the Fisher's exact test, Pearson's chisquared test or Mann-Whitney test. The results are reported as the means. Categorical and quantitative variables were dichotomized, grouped and presented as percentages or frequencies. A $p$-value $<.05$ was considered statistically significant, and a $95 \%$ confidence interval was used for the estimates.

\section{RESULTS}

Forty-six women consented to participate in this study. Six participants were withdrawn after randomization for the following reasons: two were referred to another hospital, one had re-surgery, two withdrew their informed consent and one had her surgery moved forward, causing her to miss the scheduled meeting for individual information and dialogue, and hence she was not provided a diary upon admission. Baseline data included 40 participants, 20 in the SG and 20 in the CG. The flowchart of the study is presented in Figure 1.

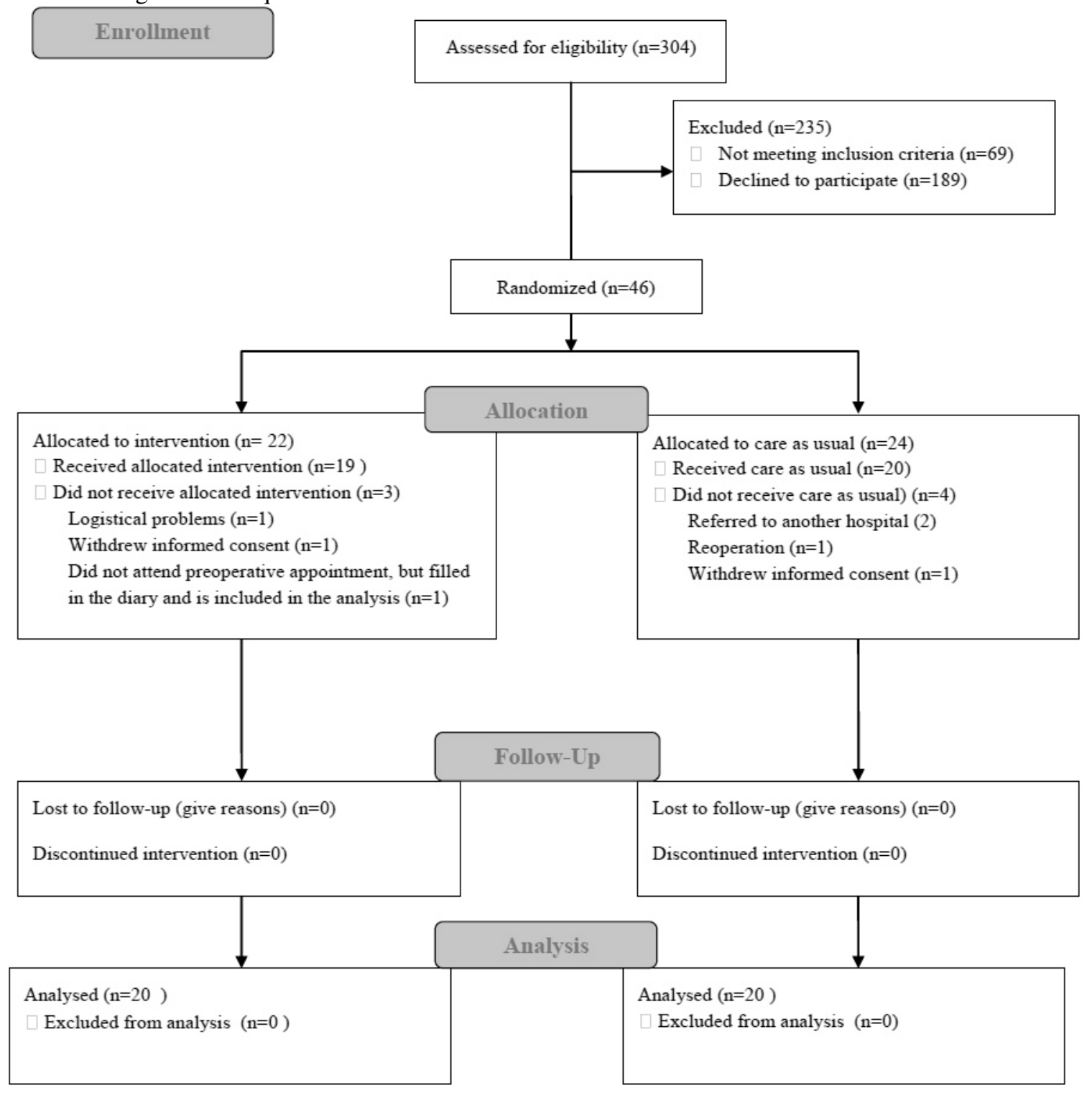

Figure 1. Participants' flow through the study 
The protocol of the study was violated regarding sample size. Due to a prolonged inclusion period, the authors decided to terminate the study on June 30, 2016, regardless of the number participants included.

The hypothesis was that preoperative structured information and dialogue decreases the level of postoperative pain and anxiety.

There were no differences between the groups on baseline characteristics, except for the type of surgery and the anesthetic technique. Anesthetic technique depends on the type of surgery. Baseline characteristics are presented in Table 1. At $6 \mathrm{~h}$ postoperatively, $95 \%$ of the participants in the SG had a VAS pain score $<6$ compared to $90 \%$ in the $\mathrm{CG}$, and at 24 $\mathrm{h}$ postoperatively, $85 \%$ in the SG had a VAS pain score $<6$ compared to $95 \%$ in the $\mathrm{CG}$.

There were no significant differences between the SG and the CG with regards to STAI anxiety scores, VAS pain scores or PPI pain scores at each time point. Controlling for type of surgery and anesthetic technique in the analysis did not change the fact that there were no differences between the groups. The results are shown in Table 2.

There were no significant differences between the SG and the CG with regards to mean morphine intake $(83 \mathrm{mg}, 95 \%$ confidence interval $[\mathrm{CI}]=[52-115]$ vs. $94 \mathrm{mg} 95 \% \mathrm{CI}[51-$ $137], p=.7$ ) and length of stay (26 hours, 95\% CI [18-34] vs. 27 hours, 95\% CI [19-34], $p=.8)$.

In the SG, 7 patients vomited within $6 \mathrm{~h}$ postoperatively com- pared to 3 in the CG, and from 6-24 h postoperatively, 4 and 5 patients in the SG and CG vomited, respectively. There were no differences in the level of nausea, oral intake and activity between the groups.

Table 1. Baseline demographic and clinical characteristics

\begin{tabular}{lll}
\hline $\begin{array}{l}\text { Demographic and Clinical } \\
\text { Characteristics }\end{array}$ & $\begin{array}{l}\text { Intervention } \\
(\mathbf{n}=20)\end{array}$ & $\begin{array}{l}\text { Control (n= 20) } \\
\text { Clinical } \\
\text { Characteristics }\end{array}$ \\
\hline $\begin{array}{l}\text { Age, years } \\
\text { Body mass index }\end{array}$ & $51( \pm 10)$ & $48( \pm 13)$ \\
ASA status & $26( \pm 4)$ & $24( \pm 4)$ \\
I & $12(60)$ & $10(50)$ \\
II & $8(40)$ & $10(50)$ \\
Charlson Comorbidity Index & $0.9( \pm 1.1)$ & $0.6( \pm 1.1)$ \\
Preoperative dexamethasone & $19(95)$ & $20(100)$ \\
Type of surgery & & \\
Abdominal & $10(50)$ & $5(25)$ \\
Laparoscopic & $6(30)$ & $12(60)$ \\
Vaginal & $4(20)$ & $3(15)$ \\
Anesthetic technique & & $12(60)$ \\
General anesthesia (GA) & $9(45)$ & $5(30)$ \\
Epidural and GA & $10(50)$ & $3(15)$ \\
Spinal & $1(5)$ & $15(75)$ \\
Intraoperative antiemetic given & & \\
Ondansetron 4 mg & $15(75)$ & $3.5( \pm 5.9)$ \\
Postoperative antiemetic given & & $1.0( \pm 4.5)$ \\
Metoclopramide oral (mg) & $2.5( \pm 7.2)$ & $1.5( \pm 2.1)$ \\
Metoclopramide sup (mg) & $1.0( \pm 4.5)$ & $99( \pm 35)$ \\
Ondansetron iv (mg) & $1.3( \pm 3.2)$ \\
Ondansetron oral (mg) & $3.0( \pm 6.1)$ & $89( \pm 39)$ \\
Duration of operation (min) & $8.7)$ \\
\hline Not. Data & \\
\hline
\end{tabular}

Note. Data are the means ( \pm SD) or numbers (\%).

Table 2. Level of anxiety and postoperative pain

\begin{tabular}{llll}
\hline Outcome & Intervention $(\mathbf{n}=\mathbf{2 0})$ & Control $(\mathbf{n}=\mathbf{2 0})$ & $\boldsymbol{p}$-value \\
\hline STAI score & & & \\
1 h preoperative $(\mathrm{n}=40)$ & $47[45-49]$ & $46[44-48]$ & .4 \\
6 h postoperative $(\mathrm{n}=36)$ & $42[39-45]$ & $42[40-44]$ & .9 \\
24 h postoperative $(\mathrm{n}=38)$ & $43[40-46]$ & $41[37-44]$ & .4 \\
Pain score & & & \\
VAS 6 $\mathrm{h}(\mathrm{n}=40)$ & $2.6[1.7-3.4]$ & $3.0[2.1-3.8]$ & .5 \\
VAS 24 $\mathrm{h}(\mathrm{n}=39)$ & $2.8[1.5-4.0]$ & $2.7[1.7-3.8]$ & .8 \\
VAS 4 weeks $(\mathrm{n}=36)$ & $1.2[0.4-2.1]$ & $1.1[0.4-1.8]$ & .8 \\
\hline
\end{tabular}

Note. Because of missing values, $\mathrm{n}$ varies slightly between the individual parameters. Data are means (95\% confidence interval). Mann Whitney U-test for comparing means for continuous variables.

\section{Discussion}

The reason for choosing a randomized controlled trial design is that the characteristics of the patients in each intervention are purely left to chance in the allocation process. However, in this study, there appears to have been an imbalance between groups in the type of surgery and anesthetic tech- nique, and this imbalance may have influenced and biased the outcome. The imbalance in the anesthetic technique can be ascribed to the imbalance in the type of surgery, as different types of surgery require different types of anesthetic techniques. In the SG, there were $25 \%$ more patients undergoing abdominal hysterectomy compared with the CG. It is 
well-known that abdominal hysterectomy causes more postoperative pain than vaginal and laparoscopic hysterectomy; hence, the primary outcome, the VAS pain score, could be biased. However, controlling for the type of surgery and anesthetic technique did not change the results.

To reduce bias and achieve balance in the allocation of participants to the SG and CG, we used block randomization; however, the randomization did not succeed perfectly. As mentioned before, there was an imbalance in the type of surgery. This imbalance may have been accidental. On the other hand, using block randomization in a non-blinded study, such as the current study, may also be a limitation, as the allocation may be predictable. However, we do not believe that this limitation has influenced the results.

The data were collected by the first author, who knew the randomization status of the patients. However, the majority of measures were subjective assessments such as the SF-MPQ, STAI and VAS, which were completed by the participants themselves.

In this randomized controlled study comparing preoperative individual information and dialogue with standard information, we found no significant differences in STAI anxiety scores, VAS pain scores and PPI pain scores. The reason for not being able to demonstrate that individual information and dialogue lowered the level of anxiety and reduced postoperative pain may be that the size of the study sample was too small and hence may have failed to support the hypothesis due to a type II error.

In the results section, we observed that more participants in the SG had moderate to severe pain at $24 \mathrm{~h}$ compared to the CG. This finding could be explained by the greater number of abdominal hysterectomies in the SG.

Recruitment was a limitation of the study. The fact that the participants may have had to come to the hospital for an additional visit was a drawback. The current study did not succeed in including the appropriate number of participants, and hence, the protocol was violated. One of the reasons for not being able to include the appropriate number of participants is that the number of hysterectomies performed at the Department of Gynecology and Obstetrics was reduced by half during the inclusion period.

Due to improved anesthetic and surgical techniques in recent decades, the length of hospitalization has been significantly reduced. Simultaneously, there has been an increase in the use of minimally invasive surgery and ambulatory surgery, in which patients are admitted on the day of the surgery. All these improvements have reduced the opportunity to give patients all the preoperative information necessary to reduce anxiety.

Despite the fact that there are no similar studies in the literature (based on gynecological patients), evidence exists that the effectiveness of preoperative interventions may lead to positive postoperative outcomes in surgical patients. ${ }^{[13,19-21]}$ However, the review by $\mathrm{Guo}^{[19]}$ that examined the effect of preoperative education interventions to reduce anxiety and improve recovery among cardiac patients produced conflicting results. Six studies were included in the review, and four of these studies measured anxiety levels. Two of the studies showed that preoperative intervention could reduce patients' level of anxiety, while two studies showed no difference in anxiety levels between the study group and the control group, which supports the results in the current study.

Only two studies in the aforementioned review ${ }^{[19]}$ examined pain as an outcome. One measured postoperative pain using the SF-MPQ and found no difference between the groups. The other study measured pain 6 months after surgery using a VAS. This study also failed to prove that a preoperative education intervention could reduce pain. Both studies support the findings of the current study.

According to the reviews by McDonald et al. ${ }^{[21]}$ and Ramesh et al., ${ }^{[20]}$ there was no statistically significant difference between the groups with regards to postoperative pain. However, as mentioned before there was a statistically significant difference in the level of anxiety. These reviews support the findings of no statistically significant difference in postoperative pain in the current study but do not support the findings of no statistically significant difference in the level of anxiety.

When we compare this study's results with the results in the study by Lee et al., ${ }^{[13]}$ we see approximately the same level of pre- and postoperative anxiety in both SG. However, when we look at the CG, we see that Lee et al. ${ }^{[13]}$ managed to demonstrate a significantly higher level of pre- and postoperative anxiety level compared to the level in the current study and they also managed to demonstrate a significantly lower level of postoperative pain. Although Lee et al. ${ }^{[13]}$ used same methods in measuring results and used some of the same time point as in the current study; the interventions in the studies were different. In the current study, the intervention took place approximately two weeks before the surgery whereas the study by Lee et al. ${ }^{[13]}$ took place the day before the surgery. In both studies, the participants were given individual intervention; however, in the study by Lee et al. ${ }^{[13]}$ the participants also got a booklet with extensive information and pictures. The difference in design may explain the difference in results. 


\section{CONClusion}

As mentioned before, we failed to demonstrate that individual information and dialog could reduce the level of preoperative anxiety and hence reduce postoperative pain among women who underwent hysterectomy. One possible reason is that we did not stratify the participants according to their level of anxiety. It might be that only those with a high level of anxiety may benefit from individual dialog and information.

\section{Implications for nursing education, practice and re- search}

In the future, it may be beneficial for patients with an elevated level of preoperative anxiety if nurses are more aware of potential symptoms of preoperative anxiety and that they have the expertise and skills to delivering knowledge-based care for those patients who have an elevated level of preoperative anxiety. If nurses are provided with education on how to identify anxiety and how to act on it, they will be able to identify patients with an elevated level of preoperative anxiety and provide proper care and hence the patients will experience reduced postoperative pain.

There is a need to conduct well-designed trials to provide evidence of whether or not preoperative interventions are effective in reducing anxiety levels and hence reduce the level of postoperative pain after hysterectomy.

\section{CONFlicts OF INTEREST Disclosure}

Both authors declare no conflict of interest.

\section{REFERENCES}

[1] Nigussie S, Belachew T, Wolancho W. Predictors of preoperative anxiety among surgical patients in Jimma University specialized teaching hospital, South Western Ethiopia. BMC Surg. 2014; 14(1): 67. PMid:25189274 https ://doi .org/10.1186/1471-2482-1 4-67

[2] Pinar G, Kurt A, Gungor T. The efficacy of preopoerative instruction in reducing anxiety following gyneoncological surgery: a case control study. World J Surg Oncol. 2011; 9(1): 38. PMid:21477315 https://doi.org/10.1186/1477-7819-9-38

[3] Pritchard MJ. Managing anxiety in the elective surgical patient. Br J Nurs. 2009; 18(7): 416-9. PMid:19373185 https ://doi .org/10 $.12968 /$ bjon. 2009.18.7.41655

[4] Carr E, Brockbank K, Allen S, et al. Patterns and frequency of anxiety in women undergoing gynaecological surgery. J Clin Nurs. 2006; 15(3): 341-52. PMid:16466484 https ://doi .org/10.111 $1 / j .1365-2702.2006 .01285 . x$

[5] Uhrenfeldt L, Aagaard H, Hall EO, et al. A qualitative meta-synthesis of patients' experiences of intra- and inter-hospital transitions. J Adv Nurs. 2013; 69(8): 1678-90. PMid:23509965 https ://doi.org/ $10.1111 /$ jan. 12134

[6] Priya P, Roach EJ. Effect of pre-operative instruction on anxiety among women undergoing abdominal hysterectomy. Nurs J India. 2013; 104(6): 245-8

[7] Brandsborg B, Nikolajsen L. Chronic pain after hysterectomy. Curr Opin Anasthesiol. 2018; 31(3): 268-73. PMid:29474214 https: //doi.org/10.1097/AC0.0000000000000586

[8] Pinto PR, McIntyre T, Araújo-Soares V, et al. Psychological factors predict an unfavorable pain trajectory after hysterectomy: a prospective cohort study on chronic postsurgical pain. Pain. 2018; 159(5): 956-67. PMid:29419656 https://doi.org/10.1097/j.pain.0 000000000001170

[9] Pinto PR, McIntyre T, Almeida A, et al. The mediating role of pain catastrophizing in the relationship between presurgical anxiety and acute postsurgical pain after hysterectomy. Pain. 2012; 153(1): 21826. PMid:22115922 https ://doi .org/10.1016/j.pain. 2011. 10.020

[10] Robleda G, Sillero-Sillero A, Puig T, et al. Influence of preoperative emotional state on postoperative pain following orthopedic and trauma surgery. Rev Lat Am. 2014; 22(5): 785-91. PMid:25493674 https://doi.org/10.1590/0104-1169.0118.2481

[11] Khan RS, Ahmed K, Blakeway E, et al. Catastrophizing: a predictive factor for postoperative pain. Am J Surg. 2011; 201(1): 122-31 PMid:20832052 https://doi.org/10.1016/j.amjsurg. 2010 .02 .007

[12] Aouad MT, Kanazi GE, Malek K, et al. Predictors of postoperative pain and analgesic requirements following abdominal hysterectomy: an observational study. J Anesth. 2016; 30(1): 72-9. PMid:26499321 https://doi.org/10.1007/s00540-015-2090-0

[13] Lee C, Liu J, Lin S, et al. Effects of educational intervention on state anxiety and pain in people undergoing spinal surgery: a randomized controlled trial. Pain Manag Nurs. 2018; 19(2): 163 71. PMid:29153299 https://doi.org/10.1016/j.pmn.2017.0 8.004

[14] Wilson CJ, Mitchelson AJ, Tzeng TH, et al. Caring for the surgically anxious patient: a review of the interventions and a guide to optimizing surgical outcomes. Am J Surg. 2016; 212(1): 151-9. PMid:26138522 https://doi.org/10.1016/j.amjsurg. 2015 .03 .023

[15] Kruzik N. Benefits of preoperative education for adult elective surgery patients. AORN J. 2009; 90(3): 381-7. PMid:19735761 https://doi.org/10.1016/j.aorn.2009.06.022

[16] Lin SY, Huang HA, Lin SC, et al. The effect of an anaesthetic information video on perioperative anxiety. Eur J Anaesthesiol. 2016; 33(2): 134-9. PMid:26196527 https://doi.org/10.1097/EJA. 0000000000000307

[17] King A, Bartley J, Johanson DL, et al. Components of preoperative anxiety: A qualitative study. J Health Psychol. 2017.

[18] Sadati L, Pazouki A, Mehdizadeh A, et al. Effect of preoperative nursing visit on preoperative anxiety and postoperative complications in candidates for laparoscopic cholecystectomy: a randomized clinical trial. Scand J Caring Sci. 2013; 27(4): 994-8. PMid:23350886 https://doi.org/10.1111/scs. 12022

[19] Guo P. Preoperative education interventions to reduce anxiety and improve recovery among cardiac surgery patients: a review of randomised controlled trials. J Clin Nurs. 2015; 24(1-2): 34-46. PMid:24894181 https://doi.org/10.1111/jocn.12618

[20] Ramesh C, Nayak BS, Pai VB, et al. Effect of Preoperative Education on Postoperative Outcomes Among Patients Undergoing Cardiac 
Surgery: A Systematic Review and Meta-Analysis. J Perianesth Nurs. 2017; 32(6): 518-29. PMid:29157759 https ://doi .org/10.101 6/j.jopan.2016.11.011

[21] McDonald S, Page MJ, Beringer K, et al. Preoperative education for hip or knee replacement. Cochrane Database Syst Rev. 2014(5). https://doi.org/10.1002/14651858.CD003526.pub3

[22] Julian LJ. Measures of anxiety: State-Trait Anxiety Inventory (STAI), Beck Anxiety Inventory (BAI), and Hospital Anxiety and Depression Scale-Anxiety (HADS-A). Arthritis Care Res. 2011; 63(S11): S46772. PMid:22588767 https://doi.org/10.1002/acr. 20561
[23] Perkins F, Werner M, Persson F, et al. Development and validation of a brief, descriptive Danish pain questionnaire (BDDPQ). Acta Anaesthesiol Scand. 2004; 48(4): 486-90. PMid:15025613 https://doi.org/10.1111/j.0001-5172.2004.00344.x

[24] Burckhardt CS, Jones KD. Adult measures of pain: the McGill Pain Questionnaire (MPQ), Rheumatoid Arthritis Pain Scale (RAPS), Short-Form McGill Pain Questionnaire (SF-MPQ), Verbal Descriptive Scale (VDS), Visual Analog Scale (VAS), and West Haven-Yale Multidisciplinary Pain Inventory (WHYMPI). Arthritis Care Res. 2003; 49(S5): S96-104. https ://doi.org/10.1002/art. 11440 